\title{
Meter-scale Flexible Touch Sensor Using Projection Capacitive Measurement Technique and Fabric Electrode for Human Position Detection
}

\author{
Seiichi Takamatsu, ${ }^{1,2^{*}}$ Takahiro Yamashita, ${ }^{2}$ Tetsuhiko Murakami, ${ }^{3}$ \\ Atsuji Masuda, ${ }^{3}$ and Toshihiro Itoh ${ }^{1,2}$ \\ ${ }^{1}$ Graduate School of Frontier Sciences, The University of Tokyo, \\ 5-1-5 Kashiwanoha, Kashiwa, Chiba 277-8561, Japan \\ ${ }^{2}$ National Institute of Advanced Industrial Science and Technology, \\ 1-2-1 Namiki, Tsukuba, Ibaraki 305-8564, Japan \\ ${ }^{3}$ Industrial Technology Center of Fukui Prefecture, \\ 10 Kitainadam 61 Kawai-washizukamatchi, Fukui, Fukui 910-0102, Japan \\ (Received October 30, 2018; accepted December 14, 2018)
}

Keywords: meter scale, touch sensor, human position sensor, conductive polymer, fabric

We developed a meter-scale large-area bendable touch sensor that utilizes a 1-m-wide fabric electrode and a projection capacitance measurement method. This sensor is used to detect human position on floors or soft beds. A conductive polymer, poly(3,4-ethylenedioxythiophene):poly(styrenesulfonate) (PEDOT:PSS), is coated on hundredmeter-long nylon fibers through a die coating technique, and the resultant fibers are woven in a weft and warp pattern with a 1-m-wide automatic looming machine, forming a 1-m-wide fabric sensor. The mechanism of sensing human position is based on the detection of the capacitance change between a conductive polymer-coated sensing fiber and a human foot. A change of approximately $3.3 \mathrm{pF}$ is measured when the fabric sensor is pushed with a human-foot-sized electrode, i.e., $6.3 \mathrm{~cm}$, under an average foot pressure of $2.6 \mathrm{~N} / \mathrm{cm}^{2}$, which is large enough to detect with conventional capacitance measurement circuits in microcontroller units (MCUs). The sensing fibers have high mechanical flexibility as they withstand tens of thousands of bending tests at a bending radius of $10 \mathrm{~mm}$. Finally, a $1 \times 1 \mathrm{~m}^{2}$ fabric sensor is connected to capacitance measurement circuits and personal computers (PCs) to construct a human position sensor system. The fabric touch sensor system demonstrates the capability to detect human positions not only on the flat surface of a floor but also on the bendable surface of a soft bed. This technology should lead to bed and floor sensors that monitor elderly people in nursing homes and hospitals.

\section{Introduction}

Our fabric touch sensors are developed to meet the following three requirements for human position sensors on flexible beds and floors: meter-scale large area, low spatial resolution, and

*Corresponding author: e-mail: seiichi-takamatsu@edu.k.u-tokyo.ac.jp

https://doi.org/10.18494/SAM.2018.2173 
high flexibility compared with existing touch and pressure sensor arrays. Specifically, because such sensors have to detect the position of a large meter-scale human body, the sensor area should be able to measure objects with a size of several meters square and a spatial resolution of 6-7 cm, corresponding to the human hand or foot size. ${ }^{(1-4)}$ In addition, the sensors on a bed are bent by being walked on or pushed by the human hand and foot; thus, the sensors should sustain bending with a radius of 1 to $2 \mathrm{~cm}$, which is the curved surface of the human foot. ${ }^{(5)}$ These human position sensors are highly demanded in Japan and other advanced countries because the population of elderly people is rapidly increasing and monitoring their health is becoming a social problem. ${ }^{(6,7)}$ To solve this problem, sensors for the monitoring and care of elderly people in nursing homes and hospitals are highly demanded to reduce the cost of care and burden to caregivers. Specifically, among the sensors used for nursing and elderly care, position sensors for elderly people are critical because it is a large burden for caregivers to monitor elderly people wandering about ${ }^{(8)}$ and to check whether they have fallen from their beds..$^{(9,10)}$

To provide these specific features, problems encountered in current touch sensor technologies should be solved. For example, micro-electromechanical system (MEMS) pressure sensors ${ }^{(11,12)}$ are expensive when used for large-area devices and are brittle owing to their construction through silicon micromachining. MEMS pressure sensors have advantages of high sensitivity and small size, which are derived from their precise silicon microstructure and high-precision microfabrication process tools. ${ }^{(11,12)}$ However, the process tools are limited to the small-diameter ( 8 inches) wafers because controlling micropatterning or alignment in the photolithography process and achieving uniformity of thin-film deposition are easy on a small silicon wafer but very difficult on a large area owing to the difficulty in tuning the surrounding atmosphere, the unevenness of the wafer, and the condition of the machining tools. ${ }^{(1-13)}$ In addition, micromachining an 8 inch wafer itself is very expensive, costing tens of thousands of US dollars. Thus, MEMS sensors are not used as a whole wafer but as a chip that is cut into tens of thousands of pieces from a whole wafer, which is also cheap. ${ }^{(12,13)}$ Silicon devices are rigid and thus easily break when bent. Silicon sensors have low mechanical flexibility and thus break easily. ${ }^{(14,15)}$ Thus, MEMS devices are not suited for large-area low-cost devices for bendable applications. Recently, however, flexible organic devices have been developed through low-temperature fabrication on plastic films. ${ }^{(16-18)}$ This low-temperature process consists of thermal deposition and other deposition systems of functional organic semiconductors without high-temperature doping and annealing in silicon micromachining. However, these thermal deposition or other deposition processes for organic semiconductor materials, especially highperformance organic semiconducting materials such as pentacene and rubrene, which are deposited using thermal evaporators, ${ }^{(18,19)}$ lead to the limitation in device size to 8 inches, the same as that in silicon devices.

To solve the fabrication problem for meter-scale flexible sensors, we developed a fabric touch sensor of human position, whose fabrication process involves the die coating of a conductive polymer and weaving it using an automatic looming machine. Figure 1 shows the concept of the fabric touch sensor of human position on soft beds or floors. The fabric sensor is composed of the woven conductive polymer PEDOT:PSS and insulating-polymer-coated fibers. The capacitance change between the human foot and the sensing fiber is measured in 


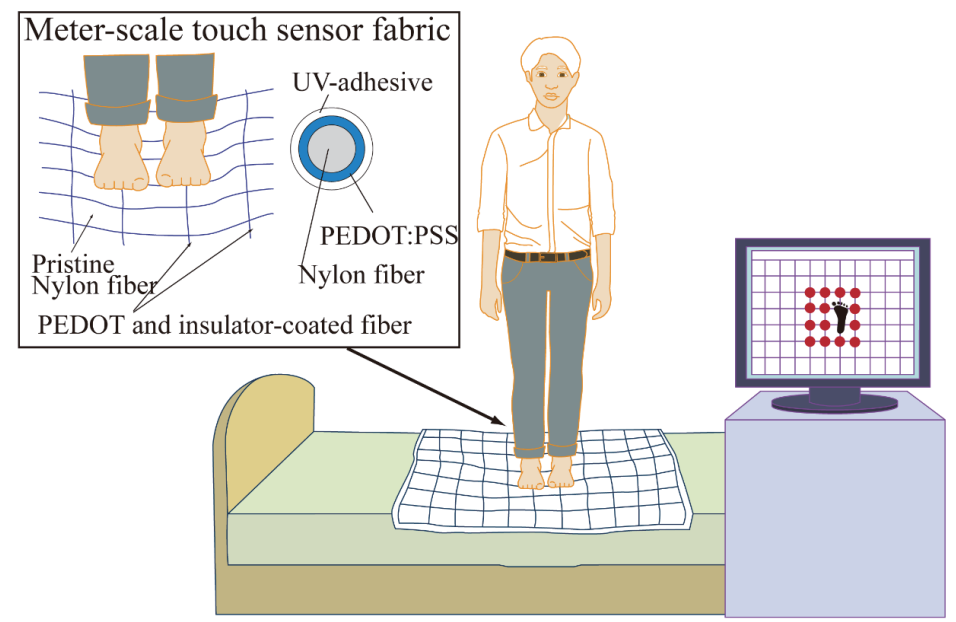

Fig. 1. (Color online) Meter-scale flexible touch sensor. Sensors are soft and flexible fabrics with conductive polymer-coated fibers. The fabric sensor detects human position by measuring the capacitance change between the fibers and the feet.

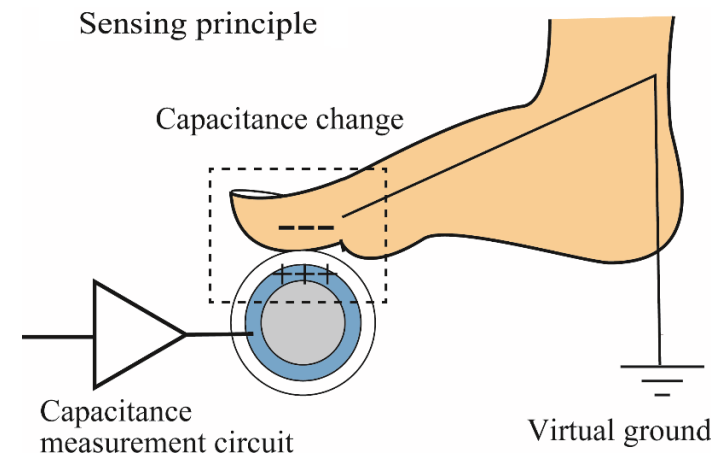

(a)

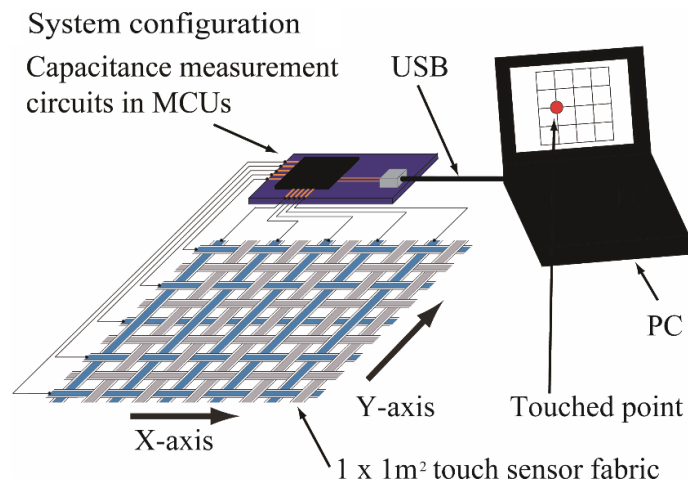

(b)

Fig. 2. (Color online) (a) Touch sensing principle. The sensors detect the capacitance change between the sensing fibers and the human feet. (b) The touch sensor system consists of a fabric sensor, capacitance measurement circuits in MCUs, and a PC for displaying human position.

order to detect human position [Fig. 2(a)]. Because a 1-m-wide meter-scale automatic looming machine is used, a meter-scale fabric sensor is easily fabricated at a low cost, as shown in Fig. 3. In addition, organic conductive polymer electrodes are inexpensive and highly flexible, enabling their application to bendable, soft surfaces. The features of the meter-scale automatic looming machine and the application of pressure sensors are reported in our previous work. ${ }^{(20)}$ On the other hand, the large-area touch sensor is demonstrated to detect human position in this study. The meter-scale sensor fabric is characterized by capacitive sensitivity and flexibility. The sensor system [Fig. 2(b)] comprises a woven fabric with conductive polymer-coated fibers, capacitance meters in conventional microcontroller units (MCUs), and personal computers (PCs). Finally, human position sensing using our system was demonstrated not only on a flat floor but also on a soft bed. 


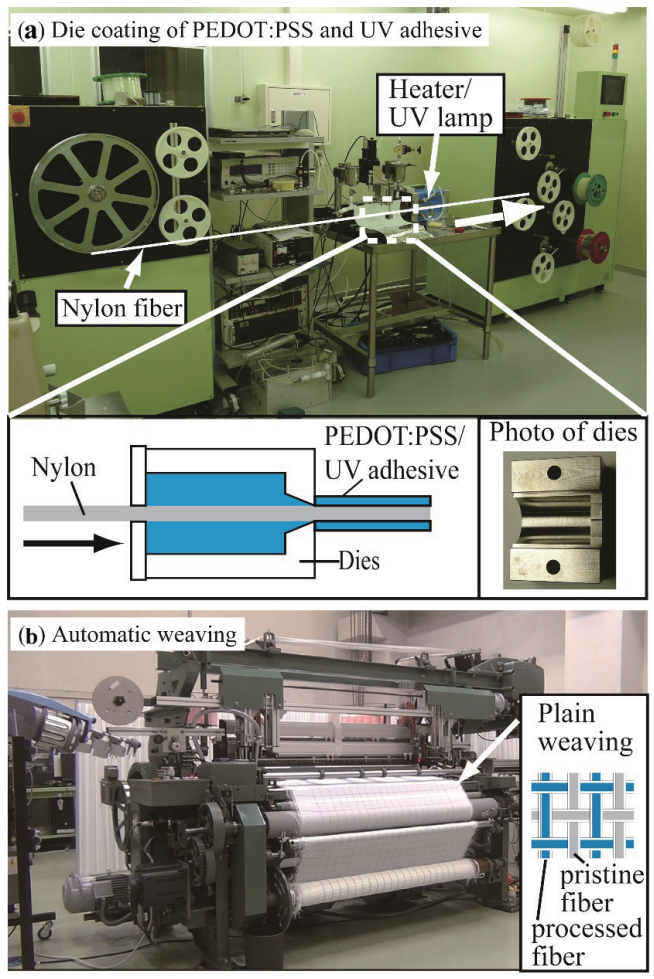

Fig. 3. (Color online) Fabrication process for meter-scale flexible touch sensor fabric. (a) The conductive and insulating polymers are coated on nylon fibers through a die coating technique. (b) The resultant fibers are woven with meter-scale automatic weaving machines in the plain weaving manner.

\section{Structure and Fabrication Process for Meter-scale Touch Sensor Fabric}

\subsection{Sensor structure and sensor system configuration}

The sensing fibers used are $485-\mu \mathrm{m}$-diameter nylon fibers coated with a conductive polymer, poly(3,4-ethylenedioxythiophene):poly(styrenesulfonate) (PEDOT:PSS) (H.C. Stark, Clevios PH1000), and an insulating polymer, i.e., an ultraviolet (UV)-curable polymer (Tesk Corp., A-1864). Such fibers are woven in a weft and warp pattern at a pitch of $5 \mathrm{~cm}$, which is defined in accordance with the average human foot width of $6.3 \mathrm{~cm} .{ }^{(10)}$ The sensor fiber should be woven at a pitch smaller than the human foot width in order to detect the foot. In addition, the rest of the conductive polymer-coated fibers are pristine polyester fibers with a diameter of 200 $\mu \mathrm{m}$. The conductive polymer-coated fibers in the sensor fabric are connected to a capacitance meter composed of conventional MCUs (C8051F700 DK, Silicon Laboratories). The insulation layer is peeled off using a cutter knife, and 5556/5558 cable connectors (5556TLS, Misumi) are attached to each fiber. Then, PEDOT:PSS is poured onto the connectors and dried to provide an electrical connection between the connectors and the electrodes on the fibers. The constructed connectors are connected to the pin of the capacitance circuit with laminated cables. The observed capacitances on the $x$ - and $y$-axis fibers are transferred to a PC through a USB cable 
with an MCU and a USB-to-UART bridge controller device (CP2102, Silicon Laboratories). The point where the $x$ - and $y$-axis fibers, which detect the output signal of the sensor electrode, cross is displayed as the location of a human body. These data are processed on the PC using the software we programmed, which is coded as a Visual Basic program (Visual Basic 2008, Microsoft).

\subsection{Human position sensing principle}

The capacitive measurement between fibers and the human foot is based on the human sensing mechanism because the human body is conductive and works as an electrode. ${ }^{(14)}$ As shown in Fig. 2(a), oscillating voltage is applied between the sensor fiber and the human body, which work as the electrode and virtual ground, respectively. The transient current flows to charge the capacitor between the sensor fibers and the human body. According to the oscillating voltage and resultant current, the capacitance is estimated. Therefore, if the human hand or human foot has sweat, a larger capacitance can be observed. In addition, if a human wears thick gloves or socks, the output capacitance becomes extremely small owing to the large gap between the sensor fibers and the human body. When a person walks on the conductive fiber fabric, the capacitance between the fibers and the foot increases. By detecting this capacitance change, the position of the human is determined. The characteristics of the fabric human position sensing system are based on the requirements described above. Finally, the human position sensor system is constructed and demonstrated.

\subsection{Fabrication by die coating and weaving}

The meter-scale touch sensor fabric is processed in two steps: coating the conductive polymer on hundred-meter-long fibers and weaving with a 1-m-wide automatic looming machine. The fabrication process starts with the coating of the conductive polymer PEDOT:PSS on a $485-\mu \mathrm{m}-$ diameter nylon fiber. The coating is performed using a die coating system, which has a die that tunes the gap between the fiber and the dies and coats the conductive polymer-water dispersion layer with the thickness of the gap between the fiber and the die. The thickness of the PEDOT:PSS film is approximately $1 \mu \mathrm{m}$. The electric resistance of the 1-m-long fiber is approximately $5-6 \mathrm{k} \Omega$, which is low enough for the conventional capacitance meter in MCUs to be used. PEDOT:PSS has the highest conductivity among conductive polymers and is flexible because it is a polymer. Second, a dielectric polymer, i.e., a UV-curable polymer, is coated on the PEDOT:PSS film as an insulating and passivating layer. Its thickness is approximately 5 $\mu \mathrm{m}$.

After coating the PEDOT:PSS and UV-curable polymer on the nylon fiber, the resultant fiber is woven with the 1-m-wide automatic looming machine. The automatic looming machine in this research was developed by our group for weaving the touch sensors. The sensor fibers are woven in a weft and warp pattern at a pitch of $5 \mathrm{~cm}$, and the rest of the parts are filled with conventional polyester fibers ( $200 \mu \mathrm{m}$ in diameter). The looming machine we developed has two apparatuses for die-coated fibers. One is a winding beam for the warp because the fibers 
have a diameter larger $(485 \mu \mathrm{m})$ than that of conventional polyester fibers $(200 \mu \mathrm{m})$ and the tensions of the polyester and large nylon fibers should be controlled separately. The other is a linear actuator for weaving warps, which can move warps between wefts without destructive friction.

\section{Basic Sensor Characteristics of Meter-scale Sensor Fabric}

\subsection{Device dimensions}

Figure 4(a) shows the meter-scale dimensions of the fabricated sensor fabric. The roll of the sensor fabric is $1.2 \mathrm{~m}$ wide and $5 \mathrm{~m}$ long. $1.2 \mathrm{~m}$ is the standard width of the fabric woven by automatic looming machines, and this width is large enough to manufacture bed sheets and carpet types of touch sensor systems. In addition to the sensor area, the sensor fabric is extremely thin (less than $0.5 \mathrm{~mm}$ ), which will lead to the fabrication of fabric sensors such as the covers of a bed and a blanket that give no discomfort to sensor users.

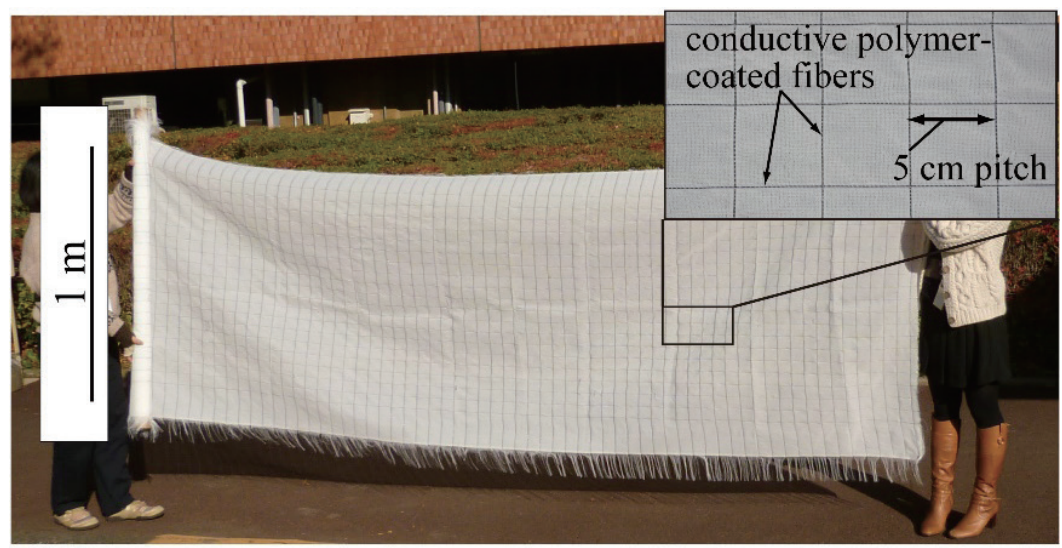

(a)

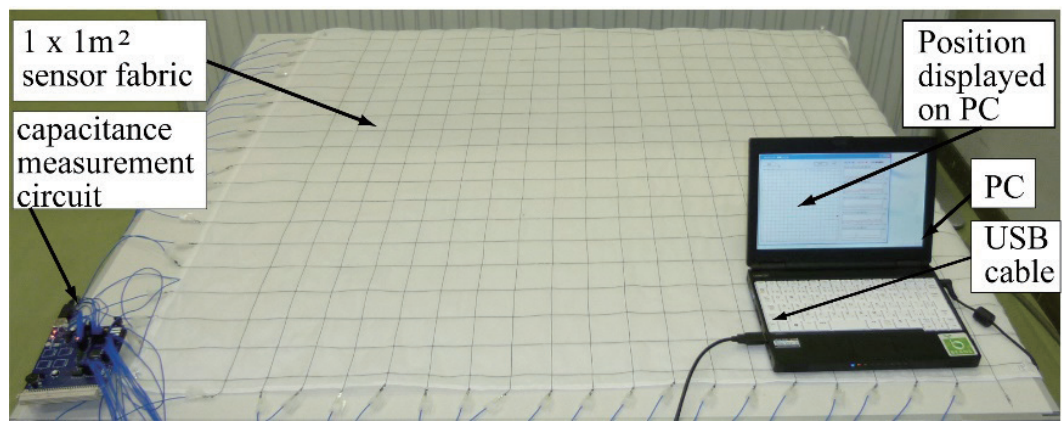

(b)

Fig. 4. (Color online) (a) Woven sensor fabric. The dimensions of the fabric are $1.2 \mathrm{~m}$ in width and $5 \mathrm{~m}$ in length. (b) The photo of the sensor system shows a $1 \times 1 \mathrm{~m}^{2}$ sensor fabric, a capacitance measurement circuit, and a PC. 


\subsection{Capacitive touch sensitivity of 1-m-long sensor fabric}

The capacitive sensitivity required to detect human feet on the sensor fabric is characterized. First, it was confirmed that the sensor can detect a human-foot-wide electrode $(6.3 \mathrm{~cm})$. Second, we examined that the sensing output at the farthermost point from the sensor amplifier was larger than the noise because the meter-long sensor is expected to have a weak output signal and contains a large noise. To confirm these two points, the basic capacitive sensitivity of our large touch sensors in terms of the applied pressure, the distance between the sensing point and the sensor amplifier from the sensor, and the size of the object for sensing were examined. The experiment was conducted with the touch sensor system as described above and a force gauge with a counter aluminum electrode, which serves as an alternative to the human foot. Specifically, the sensor fabric was first connected to a conventional capacitance measurement circuit in an MCU (C8051F700 DK, Silicon Laboratories), and the ground line was connected to the aluminum electrode. The width of the aluminum electrode is $6.3 \mathrm{~cm}$, which is the average heel width of a human foot. The pressure applied to the sensor is $2.6 \mathrm{~N} / \mathrm{cm}^{2}$, which is the average static pressure of a human foot in the case of a $60 \mathrm{~kg}$ human standing on the sensors with the contact area of $15 \times 15 \mathrm{~cm}^{2}$. The applied pressure was tuned by using a force gauge with an integrated $z$-axis automatic positioning stage (Aikoh Engineering, MODEL-FTN1-13A).

Figures 5(a) and 5(b) show the transient output response of the sensor fabric capacitance when the conductive polymer-coated fiber was pushed by a $6.3-\mathrm{cm}$-wide aluminum plate at a pressure of $2.6 \mathrm{~N} / \mathrm{cm}^{2}$. When the pushed point of the sensor fiber was $100 \mathrm{~cm}$ from the capacitance measurement circuit, the capacitance between the fiber and the electrode increased by $3.3 \mathrm{pF}$ [Fig. 5(b)]. Similarly, the capacitance increase at the nearest point from the measurement circuit was $5.6 \mathrm{pF}$. However, the signal noise was observed to be almost $0.5 \mathrm{pF}$ in both cases, as shown in Figs. 5(a) and 5(b); thus, the required capacitance change was more than $1 \mathrm{pF}$. Therefore, the capacitive output of the sensor fabric under conventional pressure and target electrode size even at $1 \mathrm{~m}$ from the amplier was large enough ( $3.3 \mathrm{pF})$ to detect even under a large noise of $0.5 \mathrm{pF}$.

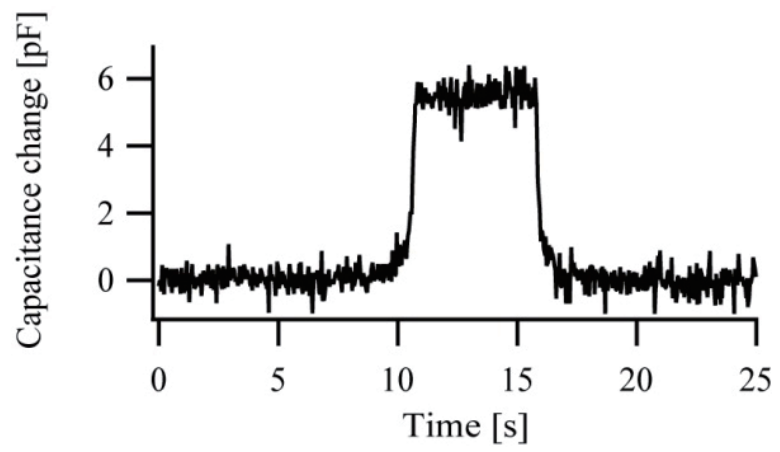

(a)

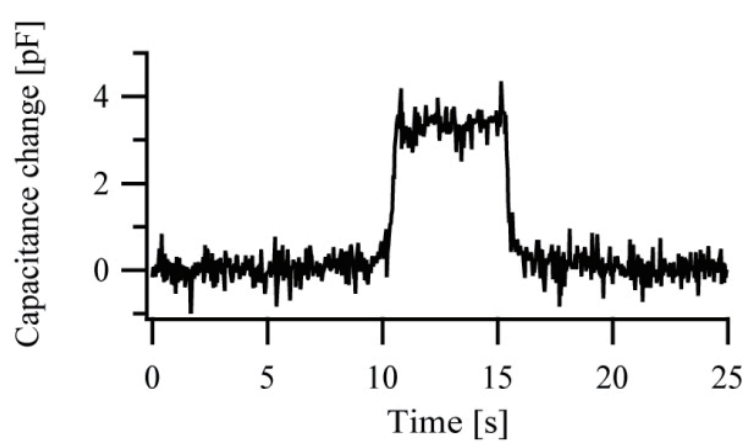

(b)

Fig. 5. Capacitance change under touch with human foot. (a) The touched point is near the capacitance measurement circuit. (b) The touched point is $1 \mathrm{~m}$ from the capacitance measurement circuit. 
The basic capacitive sensitivity of the touch sensors is as follows. First, Fig. 6 shows the relationship between the applied pressure and the resultant capacitance changes. The aluminum electrode was $6.3 \mathrm{~cm}$ wide. The capacitance changes were detected not only at the nearest point but also at the farthermost point $(100 \mathrm{~cm})$ from the amplifier. The sensor output from the sensor amplifier was relatively large even under a small input pressure of $0.1 \mathrm{~N} / \mathrm{cm}^{2}$ because the capacitance changes at the nearest and farthermost points were 2.5 and $2 \mathrm{pF}$, respectively. Thus, the sensor did not require a large input pressure in order to detect the foot. In addition, the capacitance change gradually increased with the increase in applied pressure. When the pressure was $2.6 \mathrm{~N} / \mathrm{cm}^{2}$, which is the average static pressure exerted by the human foot, the capacitance changes at the nearest and farthermost points were 5.5 and $3.3 \mathrm{pF}$, respectively, which is large enough to detect with a conventional capacitance measurement circuit as described above.

Figures 7(a) and 7(b) show the relationship between the contact area and the resultant capacitance changes. In the experiment, alternative aluminum electrodes with different lengths $(1$, $2,3,4,5,6.3,10,15,20$, and $24 \mathrm{~cm}$ ) were prepared in order to increase the contact area between the fibers and the counter electrodes. The sensing fibers were pushed by these aluminum electrodes under a pressure of $2.6 \mathrm{~N} / \mathrm{cm}^{2}$ at the nearest and farthermost points from the sensor amplifiers, and the resultant capacitance changes were measured. The capacitance changes

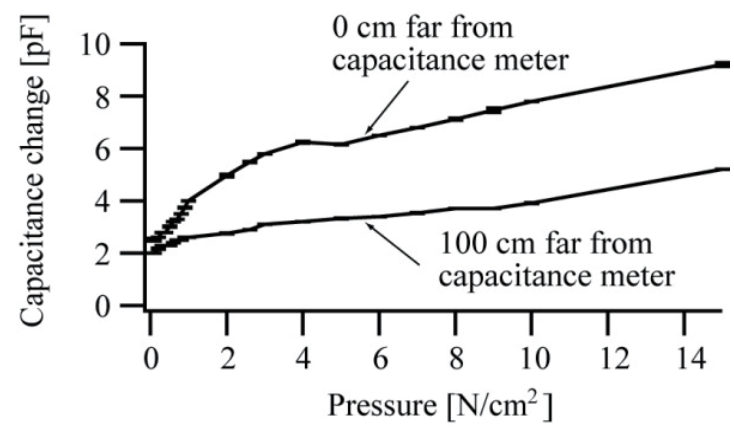

Fig. 6. Relationship between applied pressure and capacitance changes.

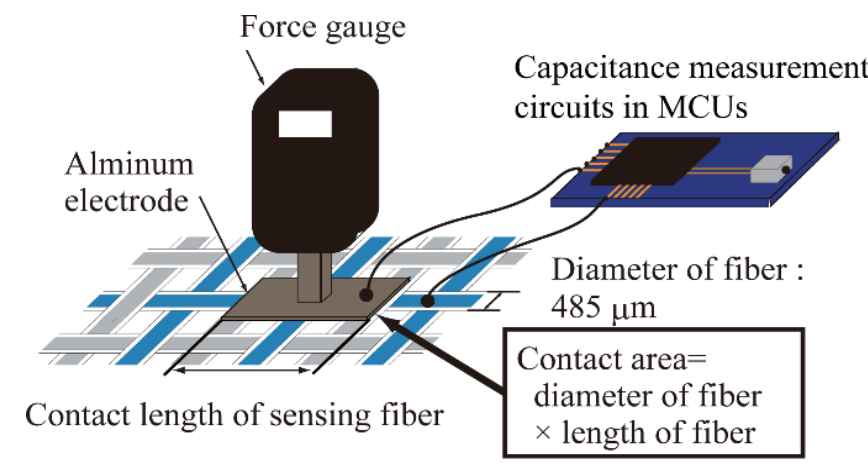

(a)

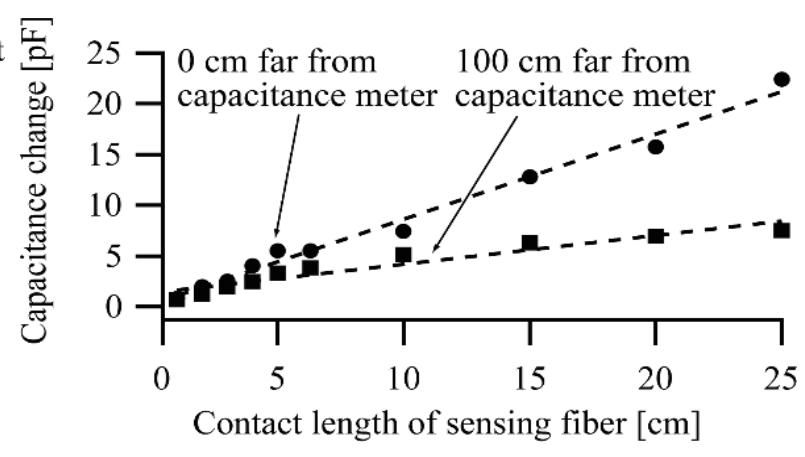

(b)

Fig. 7. (Color online) (a) Experimental setup of capacitance change of fabric touch sensors. (b) Relationship between contact length of sensing fibers and their capacitance changes. 
themselves were proportional to the contact length of the sensor fibers. This is because the contact area between the sensing fibers and the counter electrodes is defined by the length of the contact area or the length of the counter aluminum electrode, as shown in Fig. 7(a). Figure 7 (b) shows that if the contact length is $1 \mathrm{~cm}$ at the farthermost point, the capacitance change is $0.68 \mathrm{pF}$, whereas if the length is $2 \mathrm{~cm}$, the capacitance increase is $1.26 \mathrm{pF}$, which is enough for a capacitance measurement circuit. When the length was $6.3 \mathrm{~cm}$, the capacitance change was $3.3 \mathrm{pF}$. Thus, the capacitance change is proportional to the contact length of the fiber, and the length of the human foot is enough to detect the output signal with a conventional capacitance measurement circuit. Because the electric resistance of the sensor fibers is large $(>\mathrm{k} \Omega)$, the output signal is attenuated by increasing the distance from the capacitance measurement circuit to the sensing points of the fibers.

Figure 8 shows the capacitance changes when the measurement points of the sensor fibers were changed and the measurement points were $0,10,20,30,40,50,60,70,80,90$, and $100 \mathrm{~cm}$ from the capacitance measurement circuits. The applied pressure and the size of the counter aluminum electrode were $2.6 \mathrm{~N} / \mathrm{cm}^{2}$ and $2 \times 6.3 \mathrm{~cm}^{2}$, respectively. The output capacitance changes decreased when the distance between the sensor fiber and the amplifier increased. This is because the conductive polymer electrode is flexible but has high electric resistance (i.e., several $\mathrm{k} \Omega$ ), resulting in a small signal at the farthermost point. However, the smallest capacitance change was $3.3 \mathrm{pF}$, which shows that it is large enough to detect capacitance changes with a conventional capacitance measurement circuit.

Therefore, the sensor exhibited an output proportional to the applied pressure and the contact length of fibers, while the output decreased in accordance with the distance between the sensor and its amplifier circuit because of the large electric resistance of the sensor fibers. In addition, the basic sensitivity shows that even if the touched point is far from the sensor amplier (i.e., 100 $\mathrm{cm}$ far), the sensor fabric can detect more than $2 \mathrm{~cm}$ of the human body without a large applied pressure.

\subsection{Flexibility characteristics}

High flexibility is essential for fabric touch sensors because users should be able to place such sensors on the floor and bed and walk or lie on them. The mechanical properties of

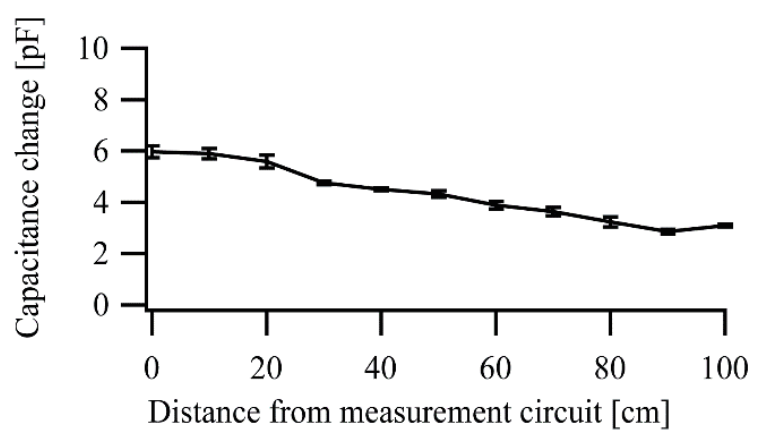

Fig. 8. Relationship between touched point distance from sensor circuit and capacitance changes. 
the fabric were characterized by cyclic bending tests. In the tests, both sides of the $25 \mathrm{~cm}$ electrodes on the fibers were connected to a multimeter (Keithley 2400), and electrical resistances were measured when the fibers were bent. For 10000 cyclic bends, both sides of the sensing electrode fibers were connected to a linear actuator (Robo-cylinder RCP3, IAI), and the fibers were bent by pushing the actuator to form a radius of $1 \mathrm{~cm}$, which is the radius of the average human foot curve. Figure 9 shows the relationship between the cycle time of the fabrics and their electrical resistance. The electrical resistance change was less than $3.8 \%$ after 10000 bends. Our touch sensor can be bent 10000 times to a radius of $1 \mathrm{~cm}$; thus, the fabric touch sensor can be used for practical applications in sensor beds and floors. In addition, because our sensor is made of fabric, the sensor can be covered on beds, sofas, and other types of furniture with complex three-dimensional surfaces.

\section{Demonstration of Human Position Sensing with Meter-scale Touch Sensors}

Finally, we demonstrated human position sensing on a soft bed and a flat floor with our constructed meter-scale touch sensor system. Our objective is the detection of human position on a soft bed with our meter-scale bendable fabric touch sensors since the previous sensors are rigid and have a small area of $30 \mathrm{~cm}^{2}$. The $1 \times 1 \mathrm{~m}^{2}$ sensor fabric, which has $19 x$-axis conductive polymer-coated fibers and $19 y$-axis ones, was connected to the capacitance meter of conventional MCUs. The measured capacitance changes of the $x$ - and $y$-axis fibers were transferred to the PC as described in Sect. 2. The crossing point of both fibers touched by a human foot was shown on the PC display as the human position. With the touch sensing software on the PC, we distinguished the human foot from noise by using the threshold capacitance change. The threshold value of the capacitance change was set at $2 \mathrm{pF}$, which is larger than the noise of the capacitance meter of the MCUs, which is approximately $0.5 \mathrm{pF}$. The point at which the fibers, whose capacitance increases are larger than the threshold of $2 \mathrm{pF}$, crosses is the point where the human foot was shown on the PC display.

In Fig. 10(a), a person walked on the sensor placed on a hard floor. The person was on the left edge of the sensor fabric, on the X4, X5, X6, and X7 fibers among the $19 x$-axis ones, and

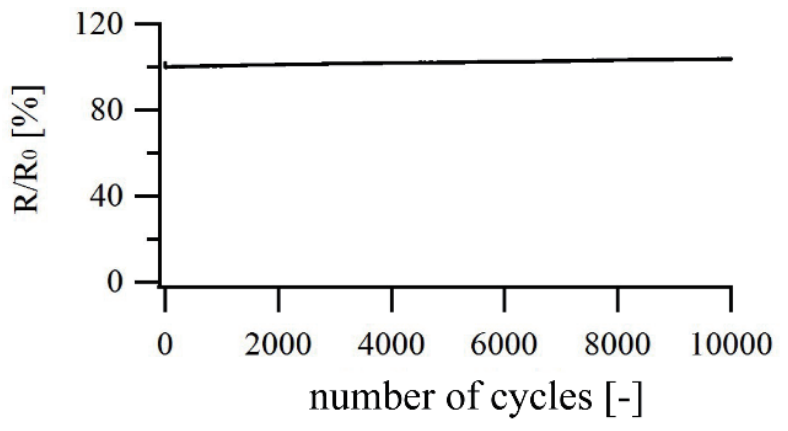

Fig. 9. Relationship between number of bending cycles of sensor fabric and electric resistance change of sensor electrode. 

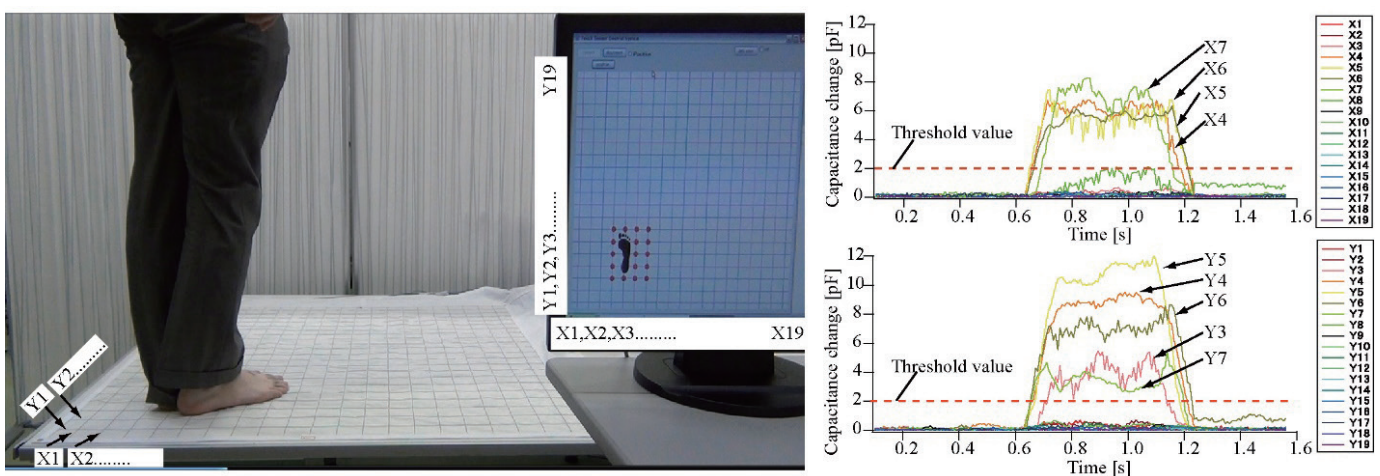

(a)
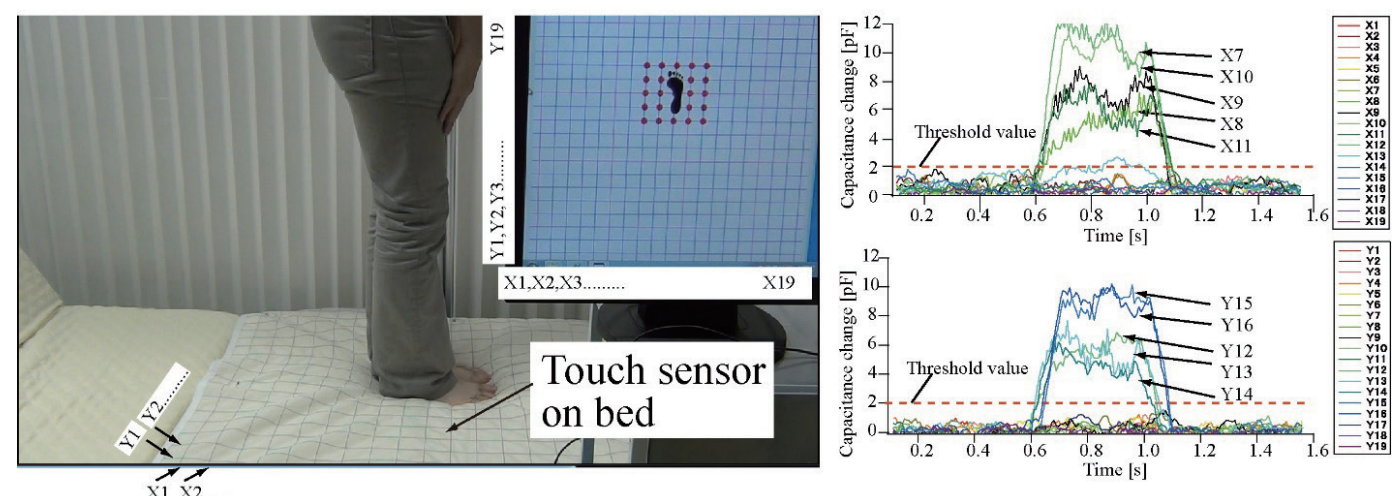

(b)

Fig. 10. (Color online) Demonstration of human position sensing. (a) Sensor fabric placed on rigid floor. (b) Fabric placed on soft bed. The sensor detects capacitance changes larger than the threshold of $2 \mathrm{pF}$.

on the Y3, Y4, Y5, Y6, and Y7 ones out of the $19 y$-axis ones. Most of the capacitance increases were more than $3.3 \mathrm{pF}$, which is larger than the threshold of $2 \mathrm{pF}$, as shown in the right part of Fig. 10(a). The red circles in the picture on the left side of Fig. 10(a) show the position of the person.

In Fig. 10(b), the person is on a soft bed on which the sensor is embedded as a sheet. Even on the bed, the shape of the fabric sensor changed by following the curvature of the foot, and the position was shown on the PC display, as shown in the left part of Fig. 10(b). The capacitance change graph showed that the X7-X11 and Y12-Y16 fibers exhibited capacitance changes of more than $2 \mathrm{pF}$. Thus, from these data, the human position was detected even on the soft bed as the sensor fabric bent and changed in shape.

Figures 11(a) and 11(b) show that a person walking can be tracked using our meter-scale sensor fabric. The motion of the person was detected by our sensors even on the bendable bed. The bending of most of rigid sensors leads to wiring fracture, but our sensor can be used even when bent because of the use of flexible conductive polymer-based sensor fibers. Thus, these results indicate that our sensor can be used for detecting the position of elderly people on large floors and soft beds in nursing homes and hospitals. 


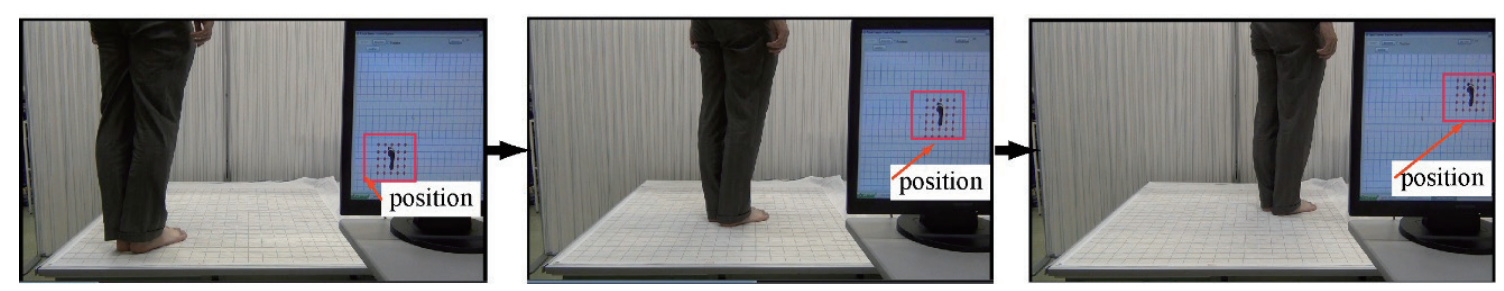

(a)

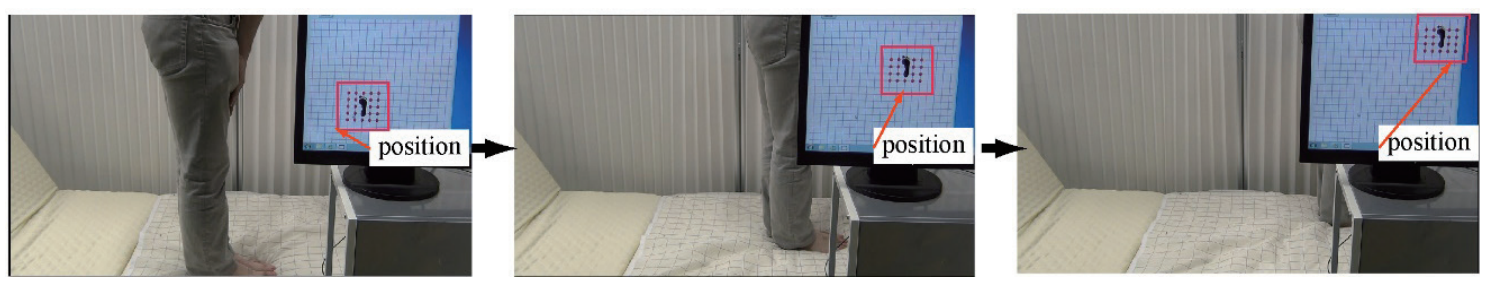

(b)

Fig. 11. (Color online) Detection of person walking. (a) Sensors on the rigid floor. (b) Sensors on the soft bed.

\section{Conclusions}

We developed a meter-scale bendable touch sensor fabric for applications to human position detection. The touch sensor fabric is a projected capacitive touch sensor consisting of woven conductive polymer fibers. A fabrication process involving the die coating of the conductive polymer and weaving with a 1-m-wide automatic looming machine was developed for forming meter-scale sensors.

The sensor fabric exhibited a capacitance increase of $3.3 \mathrm{pF}$ when pressure from a human foot (6.3-cm-wide electrode as an alternative) was placed on the sensor with an average human foot static pressure of $2.6 \mathrm{~N} / \mathrm{cm}^{2}$. The capacitance change was large enough to detect with the capacitance measurement circuit used in conventional MCUs even when the measurement point was $1 \mathrm{~m}$ far from the measurement circuit.

Even at a small pressure of $0.1 \mathrm{~N} / \mathrm{cm}^{2}$, the sensor exhibited a $2 \mathrm{pF}$ capacitance change. The sensor does not require a large pressure for detection. $3 \mathrm{pF}$ was found to be detected at a pressure of $2.6 \mathrm{~N} / \mathrm{cm}^{2}$. The meter-scale touch sensor fabric exhibited high flexibility. In cyclic bending tests, the sensor fabric was found to withstand 10000 bends to a radius of $10 \mathrm{~mm}$, resulting in only a $3.8 \%$ increase in electric resistance. Therefore, our sensor fabric is suitable for applications in flexible devices.

Finally, we demonstrated human position sensing with a $1 \times 1 \mathrm{~m}^{2}$ touch sensor system. By setting the threshold capacitance change to $2 \mathrm{pF}$, the human position was detected by our sensor system not only on a flat floor but also on a soft bed. Therefore, our large-area flexible touch sensor system will lead to applications for monitoring elderly people on large floors and beds in nursing homes and hospitals. In addition, by increasing the spatial resolution of our touch sensor array, human gait pattern during working and running will be detected. 


\section{Acknowledgments}

This research was conducted as a New Energy and Industrial Technology Development Organization (NEDO) project.

\section{References}

1 F. Viani, F. Robol, A. Polo, P. Rocca, G. Oliveri, and A. Massa: Proc. IEEE 101 (2013) 2381.

2 R. Fukui, T. Mori, and T. Sato: Adv. Rob. 26 (2012) 1127.

3 D. Ding, R. A. Cooper, P. F. Pasquina, and L. Fici-Pasquina: Maturitas 69 (2011) 131.

4 Y. Visell, A. Law, and J. Cooperstock: IEEE Trans. Haptic 2 (2009) 148.

5 Digital Human Consortium Japan (2004): Human Body Properties Database [online]. http:// www.dh.aist. go.jp/bodyDB/index-e.html (accessed January 2014).

6 A. Sixsmith and N. Johnson: IEEE Pervasive Comput. 3 (2004) 42.

7 M. Chan, E. Campo, D. Esteve, and J. Y. Fourniols: Maturitas 64 (2009) 90.

8 W. Fang, E. Stone, M. Skubic, J. M. Keller, C. Abbott, and M. Rantz: IEEE J. Biomed. Health. Inform. 17 (2013) 346.

9 H. Rimminen, J. Lindström, M. Linnavuo, and R. Sepponen: IEEE Trans. Inf. Technol. Biomed. 14 (2010) 1475.

10 N. M. Kosse, K. Brands, J. M. Bauer, T. Hortobagyi, and C. J. C. Lamoth: Int. J. Med. Inform. 82 (2013) 743.

11 M. Narducci, L. Yu-Chia, W. Fang, and J. Tsai: J. Micromech. Microeng. 23 (2013) 055007.

12 M. Esashi: Jpn. J. Appl. Phys. 51 (2012) 080001.

13 J. Lu, H. Takagi, Y. Nakano, and R. Maeda: Microsyst. Technol. 19 (2013) 775.

14 J. Lewis: Mater. Today 9 (2006) 38.

15 R. F. Cook: J. Mater. Sci. 41 (2006) 841.

16 A. Nathan, A. Ahnood, M. T. Cole, L. Carcia-Gancedo, S. Hofmann, A. J. Flewitt, M. J. Kelly, J. Robertson, W. L. Ilne, S. Lee, Y. Suzuki, P. Hiralal, G. A. J. Amaratunga, F. Bonaccorso, T. Hasan, A. C. Ferrari, A. Dyadyusha, D. Chu, S. Haque, P. Andrew, and J. Moultrie: Proc. IEEE 100 (2012) 1486.

17 T. Someya, Y. Kato, T. Sekitani, S. Iba, Y. Noguchi, Y. Murase, H. Kawaguchi, and T. Sakurai: Proc. Natl. Acad. Sci. 102 (2005) 12321.

18 A. R. Murphy and J. M. M. Frechet: Chem. Rev. 107 (2007) 1066.

19 T. Sekitani, M. Takamiya, Y. Noguchi, S. Nakano, Y. Kato, T. Sakurai, and T. Someya: Nat. Mater. 6 (2007) 413.

20 S. Takamatsu, T. Yamashita, T. Murakami, A. Masuda, and T. Itoh: Sens. Mater. 26 (2014) 559. 